\title{
Electrochemical Determination of Quercetin in Hawthorn and Onion Using a poly (L-lysine)/graphene Film Electrode
}

\author{
Xinying Ma ${ }^{1}$, Meifeng Chen ${ }^{1}$, Huiping $\mathrm{Lv}^{1}$, Zhenxu Deng ${ }^{2, *}$, Mingjing Yin ${ }^{1}$, Xueliang Wang ${ }^{1}$ \\ ${ }^{1}$ College of Chemistry and Chemical Engineering, Heze University, Shandong, Heze274015, China \\ ${ }^{2}$ College of agricultural and biological engineering, Heze University, Shandong, Heze274015, China \\ *E-mail:dengzhenxu01@126.com
}

doi: $10.20964 / 2018.07 .32$

Received: 3 March 2018 / Accepted: 6 May 2018 / Published: 5 June 2018

\begin{abstract}
A novel voltammetric method was developed for the fast determination of quercetin using apoly (Llysine)/grapheneglassy carbon electrode (PLYS/GR/GCE). Using this modified electrode as the working electrode, cyclic voltammetry $(\mathrm{CV})$ was performed on quercetin in $\mathrm{pH} 6.0$ phosphate-buffered saline (PBS). A high electrocatalytic activity was shown for the oxidation of quercetin at the PLYS/GR/GCE in this work. The oxidation of quercetin at the PLYS/GR/GCE was significantly higher than that obtained at a bare electrode. Under the optimal experimental conditions, there was a linear relationship between the oxidation peak current and the concentration of quercetin in the range of $8.1 \times 10^{-7} \sim 1.9 \times 10^{-5} \mathrm{~mol} \cdot \mathrm{L}^{-1}$ with a detection limit of $2.4 \times 10^{-9} \mathrm{~mol} \cdot \mathrm{L}^{-1}$. The method showed good reproducibility and sensitivity for the determination of quercetin. Moreover, this method was successfully applied to the determination of quercetin in hawthorn and red onion, which indicated that the PLYS/GR/GCE has promising novel applications in the detection of quercetin in real samples.
\end{abstract}

Keywords: Poly (L-lysine); Graphene; Quercetin; Electrochemical determination

\section{FULL TEXT}

(C) 2018 The Authors. Published by ESG (www.electrochemsci.org). This article is an open access article distributed under the terms and conditions of the Creative Commons Attribution license (http://creativecommons.org/licenses/by/4.0/). 